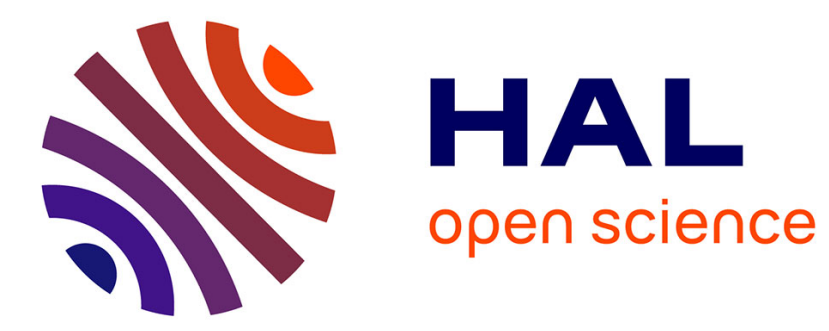

\title{
Vibration analysis of hydrogen, deuterium and tritium in metals: consequences on the isotope effect
}

\author{
Natacha Bourgeois, Jean-Claude Crivello, Pierre Cenedese, Valérie \\ Paul-Boncour, Jean-Marc Joubert
}

\section{- To cite this version:}

Natacha Bourgeois, Jean-Claude Crivello, Pierre Cenedese, Valérie Paul-Boncour, Jean-Marc Joubert. Vibration analysis of hydrogen, deuterium and tritium in metals: consequences on the isotope effect. Journal of Physics: Condensed Matter, 2018, 30 (33), pp.335402. 10.1088/1361-648X/aad259 . hal02011296

\section{HAL Id: hal-02011296 \\ https://hal.science/hal-02011296}

Submitted on 17 Mar 2021

HAL is a multi-disciplinary open access archive for the deposit and dissemination of scientific research documents, whether they are published or not. The documents may come from teaching and research institutions in France or abroad, or from public or private research centers.
L'archive ouverte pluridisciplinaire HAL, est destinée au dépôt et à la diffusion de documents scientifiques de niveau recherche, publiés ou non, émanant des établissements d'enseignement et de recherche français ou étrangers, des laboratoires publics ou privés. 


\title{
Vibration analysis of hydrogen, deuterium and tritium in metals: consequences on the isotope effect
}

\author{
Natacha Bourgeois, Jean-Claude Crivello, Pierre Cenedese, \\ Valérie Paul-Boncour, Jean-Marc Joubert \\ Université Paris Est, ICMPE (UMR 7182), CNRS, UPEC, F-94320 Thiais, France \\ E-mail: crivello@icmpe.cnrs.fr
}

March 2018

\begin{abstract}
The present study focuses on the impact of the vibrational frequencies on the thermodynamic behavior of hydrides, deuterides and tritides, using high scale harmonic phonon calculations based on first-principle calculations. $115 \mathrm{MH}_{y}$ hydrides were considered, for $y=\{0.5,1,2\}$ with $M$ among 30 metallic elements. The results were found in excellent agreement with the available experimental data and pointed out trends on the evolution of the hydride zero point energy as a function of the crystal structure and the host metal nature. Based on these information, the vibration contribution to the formation enthalpy was deduced. This contribution is responsible for the differences between the enthalpies and therefore pressures of formation of the hydride, deuteride and tritide compounds. This so-called "isotope effect" is experimentally observed but has never been studied by large scale calculations. A straightforward method has been developed allowing to quantify the isotope effect at non zero temperature. It explains the experimentally observed relative stability of hydride, deuteride and tritide compounds. As a major achievement, a new phenomenon was highlighted, which has never been anticipated, consisting in an inversion of the isotope effect when the temperature increases.
\end{abstract}

Keywords: Hydrogen and its isotopes, Phonon states and bands, Inorganic metal hydrides Submitted to: J. Phys.: Condens. Matter 


\section{Introduction}

The lattice dynamic effects are particularly important to understand the thermodynamic behavior of metal hydrides $M \mathrm{H}_{y}$. Because of the low mass of hydrogen atoms, the vibration frequencies reach values larger than $10 \mathrm{THz}$ in the optical modes. The

associated vibration energy $E_{\mathrm{vib}}^{M \mathrm{H}_{y}}$ is therefore highly destabilizing for the crystal lattice. Even at $0 \mathrm{~K}$, the corresponding vibration energy, called Zero Point Energy (ZPE), is non negligible. Therefore, determining the vibration frequencies has been the purpose of extensive theoretical and experimental studies using techniques such as inelastic neutron scattering, Raman and infrared spectroscopy [1]. The early works were based on measurements of the neutron total cross section to obtain the energy level of the optical frequencies [2]. The metal-hydrogen equilibrium distance $r$ was pointed out as a central parameter driving the vibration modes, for a given crystal structure regardless the nature of the metal. Indeed, a linear decrease of the optical frequencies with increasing $r$ was suggested [3, 1, 4].

Besides, hydride vibration modes allow to explain the important changes in the relative stability and in thermodynamic properties when substituting hydrogen by its heavier isotopes deuterium (D) and tritium $(\mathrm{T})$ in the metallic host lattice. The deuteride $M \mathrm{D}_{y}$ and the tritide $M \mathrm{~T}_{y}$ usually have the same crystal structure as the hydride. However, the vibration modes are considerably affected by the large mass differences between the three isotopes: the heavier the atom is, the lower the optical frequencies are.

This so-called isotope effect has raised considerable interest in both fundamental [5] and applied research, for applications such as hydrogen isotope separation and storage [6]. The isotope effect is commonly quantified by comparing the measured formation pressures of the hydride, deuteride and tritide. The lowest equilibrium pressure corresponds to the most stable compound, with the most negative Gibbs energy of formation. The formation enthalpies may also be measured by calorimetry or derived from equilibrium pressure data.

In the present paper, the expression "normal isotope effect" will be used when the tritide is more stable than the corresponding deuteride, itself more stable than the hydride. The equilibrium pressures are then such as: $P_{\mathrm{eq}}^{\mathrm{T}_{2}}<P_{\mathrm{eq}}^{\mathrm{D}_{2}}<P_{\mathrm{eq}}^{\mathrm{H}_{2}}$. The "inverse isotope effect" corresponds to the reverse case: $P_{\mathrm{eq}}^{\mathrm{H}_{2}}<P_{\mathrm{eq}}^{\mathrm{D}_{2}}<P_{\mathrm{eq}}^{\mathrm{T}_{2}}$. The $\mathrm{VH}_{2}$ dihydride illustrates the normal isotope effect, whereas the $\mathrm{PdH}$ hydride presents a marked inverse isotope effect.

Phonon calculations based on first-principle methods allow to determine the vibration energy of any stable or metastable compound. The advantage is twofold: first, they allow to isolate the vibration contribution from the enthalpy of formation, which is involved in the isotope effect; second, they provide data on metastable or high pressure hydrides which can hardly be synthesized. Even if metastable hydrides are not experimentally observed, their study allows to widen the range of possible comparisons between different hydride crystal structures, for a given metal. Phonon calculations are 
Table 1. List of the 12 considered crystal structure prototypes of $M \mathrm{H}_{y}$ hydrides. P.S.: Pearson Symbol, S.G.:Space Group, Wyckoff Positions, $\mathcal{I}$ environment. Supercell details for the phonon calculations: number of displacements (Dl.), size and number of atoms given from the conventional description, or primitive one if indicated by a *

\begin{tabular}{lrllllllr}
\hline Prototype & $y$ & P.S. & S.G. & Wyckoff Positions & Environment & Dl. & Size & Atoms \\
\hline $\mathrm{CaF}_{2}$ & 2 & $c F 12$ & $F m \overline{3} m(225)$ & $M: 4 a, \mathrm{H}: 8 c$ & ideal te & 2 & $2 \times 2 \times 2$ & 96 \\
$\mathrm{ZnO}$ & 1 & $h P 4$ & $P 6_{3} m c(186)$ & $M: 2 b, \mathrm{H}: 2 b$ & distorded te & 4 & $2 \times 2 \times 2$ & 32 \\
$\mathrm{PtS}$ & 1 & $t P 4$ & $P 4_{2} / m m c(131)$ & $M: 2 c, \mathrm{H}: 2 e$ & distorded te & 3 & $3 \times 3 \times 2$ & 72 \\
$\mathrm{NbH}$ & 1 & $o P 8$ & $P n n n(48)$ & $M: 4 f, \mathrm{H}: 2 a, 2 b$ & distorded te & 4 & $2 \times 2 \times 2$ & 64 \\
$\mathrm{Ta}_{2} \mathrm{H}$ & 0.5 & $o S 6$ & $C 222(21)$ & $M: 4 k, \mathrm{H}: 2 a$ & ideal te & 5 & $2 \times 2 \times 2^{*}$ & 24 \\
$\mathrm{NaCl}$ & 1 & $c F 8$ & $F m \overline{3} m(225)$ & $M: 1 a, \mathrm{H}: 1 b$ & ideal o & 2 & $2 \times 2 \times 2$ & 64 \\
$\mathrm{NiAs}$ & 1 & $h P 2$ & $P 6_{3} / m m c(194)$ & $M: 2 c, \mathrm{H}: 2 a$ & ideal o & 2 & $2 \times 2 \times 2$ & 32 \\
$\mathrm{CdI}_{2}$ & 0.5 & $h P 3$ & $P \overline{3} m 1(164)$ & $M: 2 d, \mathrm{H}: 1 a$ & ideal o & 3 & $2 \times 2 \times 2$ & 24 \\
$\mathrm{AuTe}_{2}$ & 0.5 & $m C 6$ & $C 2 / m(12)$ & $M: 4 i, \mathrm{H}: 2 a$ & distorded o & 6 & $2 \times 2 \times 2$ & 48 \\
$\mathrm{TiO}_{2}$-ana & 0.5 & $t I 12$ & $I 4_{1} / a m d(141)$ & $M: 8 e, \mathrm{H}: 4 b$ & distorded o & 3 & $2 \times 2 \times 2^{*}$ & 48 \\
$\mathrm{TiO}_{2}$ & 2 & $t P 6$ & $P 4_{2} / m n m(136)$ & $M: 2 a, \mathrm{H}: 4 f$ & distorded tr & 3 & $2 \times 2 \times 2$ & 48 \\
$\mathrm{BN}-b^{1}$ & 1 & $h P 4$ & $P 6_{3} / m m c(194)$ & $M: 2 c, \mathrm{H}: 2 d$ & ideal tr & 2 & $2 \times 2 \times 2$ & 32 \\
\hline
\end{tabular}

therefore particularly appropriate to show the effect of the crystal geometry, without changing the chemistry. However, no large scale theoretical study had been undertaken yet.

In this frame, the present study proposes massive phonon calculations within the harmonic approximation coupled with calculations based on the Density Functional Theory (DFT). It supplements a recent publication reporting high throughput calculations of binary metal hydrides [7], where the objective was to define the sequence of stable hydrides (gound-state) for a given system. For this purpose, the formation enthalpies of 30 potential crystal structures were systematically computed for 31 binary $M-\mathrm{H}$ systems. Phonon calculation was done for all the crystal structures on the groundstate within an error of $5 \mathrm{~kJ} / \mathrm{mol}$. Mechanically unstable compounds with considerable imaginary frequencies were not considered.

In the present work, a careful analysis of vibrational contribution in the ordered hydrides is made on a set of $115 M \mathcal{I}_{y}$ compounds with $y=0.5,1,2$, involving 12 different crystal structure prototypes, having a unique kind of coordination site for $\mathcal{I}$ atom among the 30 original ones. The host metals $M$ are chosen as: alkaline (Li, Na, K, Rb), alkaline earth (Mg, Ca, Sr) and transition metals (Sc, Y, Ti, Zr, Hf, V, Nb, Ta, Cr, Mo, Mn, Tc, Fe, Ru, Co, Rh, Ni, Pd, Pt, Cu, Ag, Zn, Cd). The $\mathcal{I}$ atom stands for H, D, T, i.e. hydrogen, deuterium and tritium respectively.

The 115 considered hydrides are listed in the supplementary material (SM-A). The 12 crystal structure prototypes are listed in table 1.

For each structure, the $\mathcal{I}$ atom fully occupies one site among the three coordinationtypes: tetrahedral (te), octahedral (o) and triangular (tr), as represented in figure 1. One may note that these structures are representative of the stable hydrides found in most systems, but they are not stable in all $M-\mathrm{H}$ systems. 


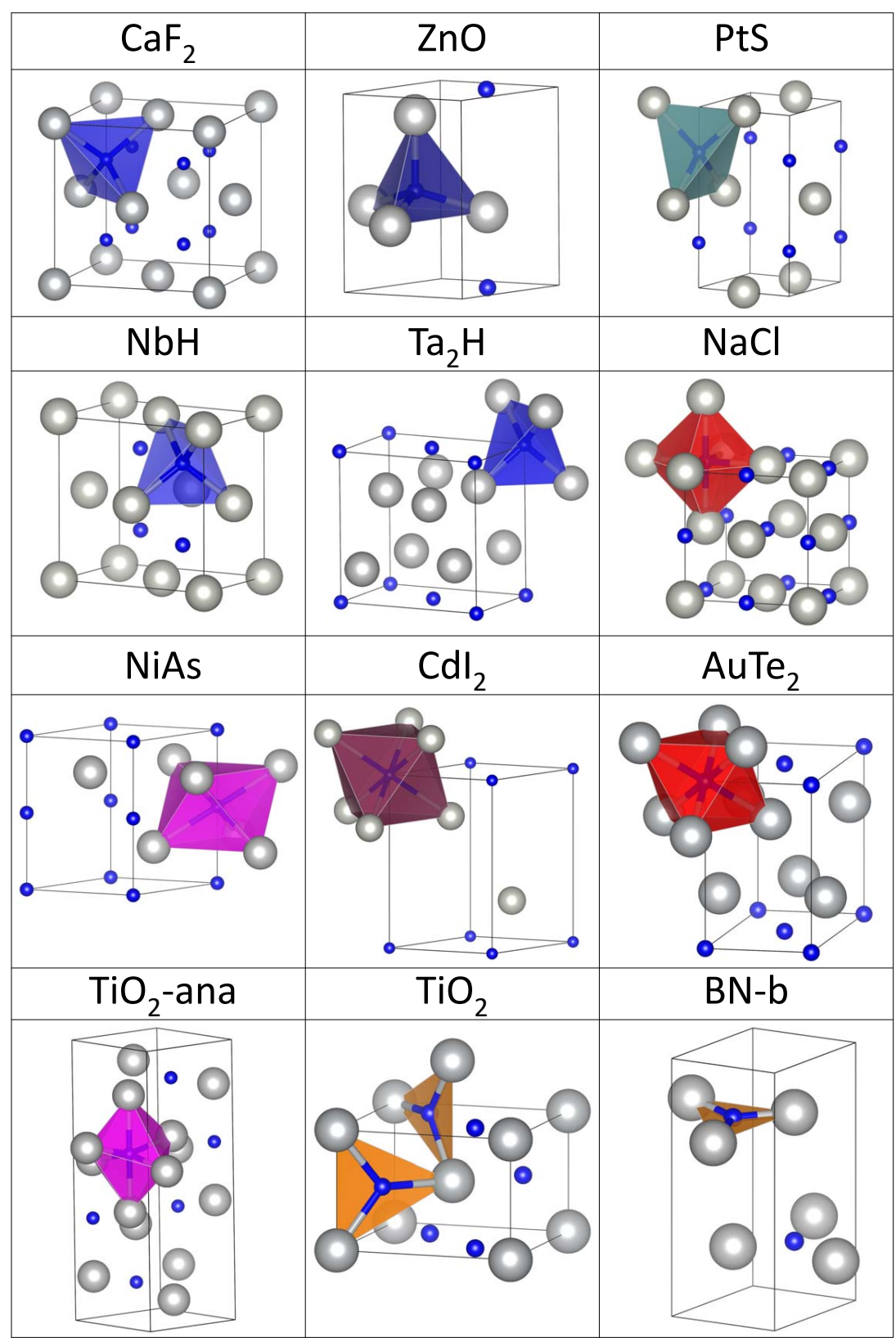

Figure 1. Crystallographic structures considered in the present study. The large gray balls represent the metal atoms and the small blue ones the hydrogen atoms. The colors identify the three different coordinations of $\mathcal{I}$ atoms tetrahedral (blue), octahedral (red) and triangular (orange). 
After the methodology description (section 2), the results will be discussed in the section 3: first, the analyses of the calculated hydride ZPE with its effect on the formation enthalpy (3.1) and, second, the predictions of the isotope effect (3.2). In each subsection, the calculated results are compared with the experimental data available in the literature.

\section{Methodology}

\subsection{Harmonic phonon calculations}

The hydride vibration modes were determined within the classical theory of harmonic lattice introduced for example in Ref. [8]. In the frame of the frozen phonons method [9], DFT calculations [10] were performed on supercells with relevant atomic displacements produced by the phonopy code [11]. Shape and size of the supercells were chosen according to the symmetry of the original phase with a minimum of $6 \AA$ as cell parameter, details are given in table 1. Anharmonic contributions have not been considered in our approach. The DFT calculations were performed using the plane-wave based Vienna Ab-initio Simulation Package (VASP) [12, 13], with a cutoff energy chosen at $800 \mathrm{eV}$. The exchange and correlation potential and energy were described within the generalized gradient approximation (GGA) using the PerdewBurke-Ernzerhof functional (PBE) [14, 15]. The Monkhorst-Pack scheme was used to sample the Brillouin zone [16], where the k-meshing number of supercell was inversely proportional to those of the original cell optimized from the previous study [7]. The lattice parameters and internal coordinates were optimized self-consistently with respect to forces according to the purely electronic calculation. In order to be able to compute energy difference at $0 \mathrm{~K}$, the dihydrogen molecule was calculated in the same condition using plane waves considering a dimer in an asymmetric box of size $11 \times 12 \times 13 \AA^{3}$. The phonopy code was used to determine the interatomic force constants from the VASP calculated interatomic forces (Hellman-Feynman theorem) [11]. The frequencies of vibration $\omega$ were summed to determine the ZPE within the quantum theory of harmonic crystals,

$$
E_{\mathrm{vib}, 0}=\sum_{\mathbf{q}, s} \frac{1}{2} \hbar \omega_{\mathbf{q}, s}
$$

with $\mathbf{q}$ the wave vector and $s$ the index of the dispersion branch. The total contribution to the energy of formation is the difference between the ZPE of the $M \mathcal{I}_{y}$ compound and the pure elements, the $\mathcal{I}_{2}$ molecule and the pure metal $M$ in its stable structure as $0 \mathrm{~K}$ :

$$
\Delta E_{\mathrm{vib}}^{M \mathcal{I}_{y}}=E_{\mathrm{vib}}^{M \mathcal{I}_{y}}-\frac{y}{2} E_{\mathrm{vib}}^{\mathcal{I}_{2}}-E_{\mathrm{vib}}^{M}
$$

The ZPE energies $E_{\mathrm{vib}}^{M \mathcal{I}_{y}}$ and $E_{\mathrm{vib}}^{\mathcal{I}_{2}}$ were computed using the mass of the $\mathcal{I}$ element $(\mathcal{I}=$ $\mathrm{H}, \mathrm{D}$ or $\mathrm{T}$ and $m_{\mathrm{H}}=1.008 \mathrm{u}, m_{\mathrm{D}}=2.014 \mathrm{u}$ or $\left.m_{\mathrm{T}}=3.016 \mathrm{u}\right)$, based on the DFT calculations performed with $\mathcal{I}=\mathrm{H}$. In the following, the energies will be given in $\mathrm{kJ} / \mathrm{mol}$ for 1 mole of compound $M \mathcal{I}_{y}$. 


\subsection{Calculations at non zero temperatures}

The temperature dependence of the energy $E_{\text {vib }}$ and entropy $S_{\text {vib }}$ of vibration of the hydrides and metals were calculated via harmonic phonon calculations within the Bose-Einstein statistic. The NIST/JANAF [17] database was used to describe the temperature dependence of $E^{\mathcal{I}_{2}}$ and $S^{\mathcal{I}_{2}}$ of the $\mathcal{I}_{2}$ molecules. The origin of the enthalpy at $0 \mathrm{~K}$, defined to within a constant, is shifted to the ZPE energies of equation (2), obtained from the phonon calculation.

\section{Results and discussion}

\subsection{Zero point energy calculations}

First, the reliability of the calculations was verified systematically comparing the frequency spectra to the experimental data. As an example, the calculated band diagrams of $\mathrm{LiD}$ were found in agreement with the inelastic neutron scattering (INS) data [18], as shown in figure 2. In addition, the phonon density of states of $\mathrm{MgH}_{2}$ agrees
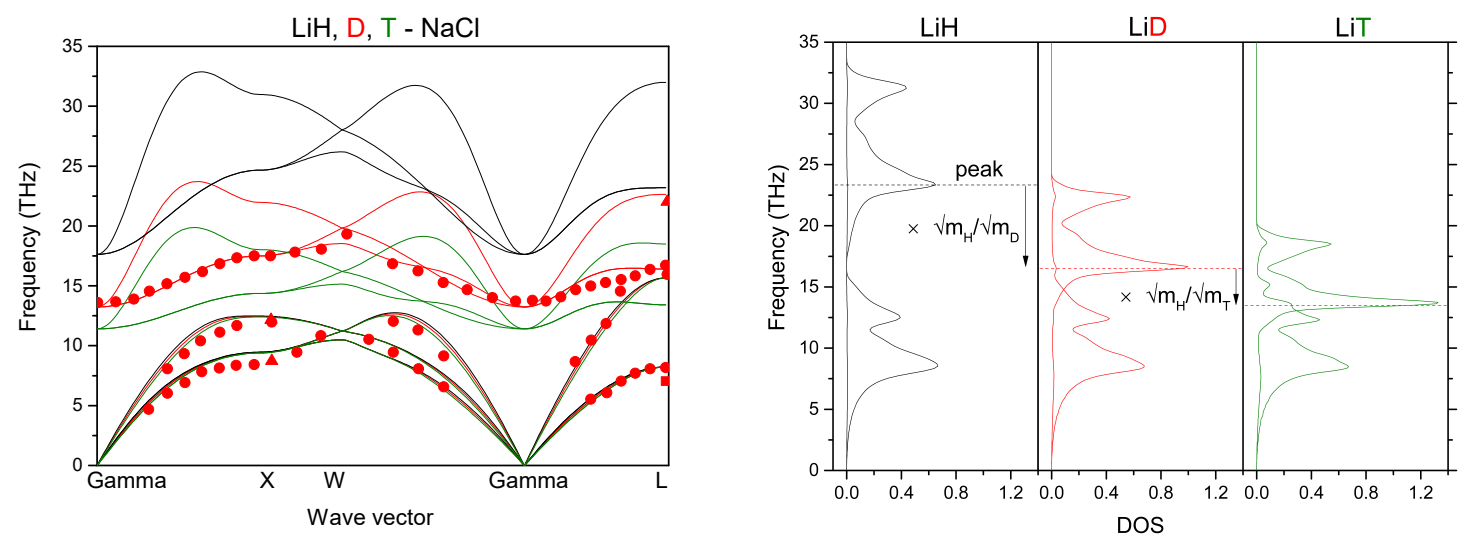

Figure 2. Calculated band diagrams (left) and partial phonon density of states (DOS) (right) of $\mathrm{LiH}, \mathrm{LiD}, \mathrm{LiT}(\mathrm{NaCl})$ represented by the solid lines, together with experimental data on $\mathrm{LiD}$ [18] represented by the symbols. The red and blue dashed lines correspond to the optical frequency peaks of the deuteride and tritide deduced from that of the hydride under the assumptions of the Einstein model.

also with the precise INS data measured at $7 \mathrm{~K}$ [19]. Besides, many lower resolution experimental data supplied only the optical frequency ranges. They are compiled in table 2, together with our calculated data. The agreement is satisfactory, except an underestimation for $\mathrm{PdH}$ hydride, due to the known important anharmonic contribution whose treatment requires specific methods [20], out of the scope of the present study, but the agreement justifies that anharmonic approximation contribution may be neglected in the other cases.

Hence, reliable ZPE values were derived from the vibration frequencies from equation (1). The ZPE, designated here by $E_{\mathrm{vib}, 0}$, was determined for the set of hydrides, for 
Table 2. Calculated (present study) and experimental optical frequency peak $(\mathrm{THz})$ measured by scattering cross section of hydrogen (SCS), inelastic neutron scattering (INS) or Fourier transform infrared spectroscopy (FTIR). The calculations are performed for ordered $M \mathrm{H}_{y}$ compounds. When the experimental measurement corresponds to a non stoichiometric compound, the value of $y$ is given.

\begin{tabular}{|c|c|c|c|c|}
\hline$M \mathrm{H}_{y}$ (prototype) & Calc. (THz) & Exp. (THz) & Meth. - compared value(s) & Ref. \\
\hline $\mathrm{CoH}(\mathrm{NaCl})$ & 23.0 & 24.7 & DIN - maximum of the first peak & {$[21]$} \\
\hline $\mathrm{CrH}(\mathrm{NiAs})$ & 31.4 & 29.5 & DIN - maximum of the main peak & {$[22]$} \\
\hline $\mathrm{CrH}(\mathrm{NiAs})$ & 31.4 & 29.3 & DIN - maximum of the main peak at $15 \mathrm{~K}$ & {$[23]$} \\
\hline $\mathrm{CuH}(\mathrm{ZnO})$ & $25.3-36.0$ & $21.6-35.4$ & IRTF - total range & {$[24]$} \\
\hline $\mathrm{FeH}(\mathrm{dhcp})$ & $26.3(\mathrm{NiAs})$ & 25.4 & DIN - maximum of the main peak at $90 \mathrm{~K}$ & {$[25]$} \\
\hline $\mathrm{LiH}(\mathrm{NaCl})$ & $23.3 / 31.2$ & $17.7 / 33.6$ & DIN - maxima of the two main peaks & {$[26]$} \\
\hline $\mathrm{MgH}_{2}\left(\mathrm{TiO}_{2}\right)$ & $30.5 / 36.1 / 42.3$ & $30.1 / 35.6 / 42.8$ & DIN - maxima of the two main peaks at $7 \mathrm{~K}$ & {$[1]$} \\
\hline $\mathrm{MnH}(\mathrm{NiAs})$ & 30.4 & 26.8 & DIN - maximum of the main peak at $100 \mathrm{~K}(y=0.86)$ & {$[27]$} \\
\hline $\mathrm{MoH}(\mathrm{NiAs})$ & 27.1 & 27.3 & DIN - maximum of the main peak at $15 \mathrm{~K}(y=1.2)$ & {$[23]$} \\
\hline $\mathrm{NbH}(\mathrm{NbH})$ & 31.4 & 36.3 & SCS - maximum of the first peak $(y=0.85)$ & {$[3]$} \\
\hline $\mathrm{NbH}_{2}\left(\mathrm{CaF}_{2}\right)$ & 36.3 & 35.8 & DIN - mean value at $80 \mathrm{~K}$ & {$[1]$} \\
\hline $\mathrm{NbH}_{2}\left(\mathrm{CaF}_{2}\right)$ & $34.3 / 37.8$ & $28.0 / 40.4$ & DIN - maxima of the two main peaks at $10 \mathrm{~K}(y=0.7)$ & {$[28]$} \\
\hline $\mathrm{NbD}_{2}\left(\mathrm{CaF}_{2}\right)$ & $24.2 / 26.8$ & $20.8 / 29.0$ & DIN - maxima of the two main peaks at $10 \mathrm{~K}(y=0.85)$ & {$[28]$} \\
\hline $\mathrm{NbT}_{2}\left(\mathrm{CaF}_{2}\right)$ & $19.9 / 21.9$ & $17.4 / 24.4$ & DIN - maxima of the two main peaks at $10 \mathrm{~K}(y=0.7)$ & {$[28]$} \\
\hline $\mathrm{NiH}(\mathrm{NaCl})$ & 19.2 & 21.5 & DIN - maximum of the main peak at $15 \mathrm{~K}(y=1.05)$ & {$[23]$} \\
\hline $\mathrm{PdH}(\mathrm{NaCl})$ & 9.7 & 13.5 & DIN - maximum of the main peak at $15 \mathrm{~K}(y=0.99)$ & {$[29]$} \\
\hline $\mathrm{TaH}_{0.5}$ (monoclinic) & $31.1 / 42.0$ & $30.0 / 42.8$ & DIN - maxima of the two main peaks & {$[30]$} \\
\hline $\mathrm{TaH}_{0.5}\left(\mathrm{Ta}_{2} \mathrm{H}\right)$ & 31.1 & 35.1 & DIN - maximum of the first peak $(y=0.7)$ & {$[3]$} \\
\hline $\mathrm{TiH}_{2}\left(\mathrm{CaF}_{2}\right)$ & 36.9 & 36.0 & DIN - mean value at $80 \mathrm{~K}$ & {$[1]$} \\
\hline $\mathrm{TiH}_{2}\left(\mathrm{CaF}_{2}\right)$ & 38.3 & 35.1 & DIN - maximum of the main peak at $300 \mathrm{~K}$ & {$[31]$} \\
\hline $\mathrm{VH}_{0.5}\left(\mathrm{AuTe}_{2}\right)$ & no peak/55.3 & $31.4 / 55.6$ & DIN - maxima of the two main peaks at $80 \mathrm{~K}$ & {$[32]$} \\
\hline $\mathrm{VD}_{0.5}\left(\mathrm{AuTe}_{2}\right)$ & no peak/39.2 & $21.0 / 39.7$ & DIN - maxima of the two main peaks at $80 \mathrm{~K}$ & {$[32]$} \\
\hline $\mathrm{VT}_{0.5}\left(\mathrm{AuTe}_{2}\right)$ & no peak/32.2 & $18.4 / 32.6$ & DIN - maxima of the two main peaks at $295 \mathrm{~K}$ & {$[32]$} \\
\hline $\mathrm{YH}_{2}\left(\mathrm{CaF}_{2}\right)$ & 30.0 & 30.7 & DIN - mean value & {$[33]$} \\
\hline $\mathrm{ZrH}_{2}\left(\mathrm{CaF}_{2}\right)$ & 34.6 & 34.2 & DIN - mean value & {$[34]$} \\
\hline
\end{tabular}

the pure metals in their reference states and for the $\mathrm{H}_{2}$ molecule with the same methodology. The ZPE values for $\mathrm{H}_{2}, \mathrm{D}_{2}$ and $\mathrm{T}_{2}$ were computed respectively at 24.9, 17.6 and $14.4 \mathrm{~kJ} / \mathrm{mol}$, each ratio corresponding to the inverse ratio of the squared masses. The agreement is satisfactory with the experimental values of Irikura [35] of 26.1 and $18.5 \mathrm{~kJ} / \mathrm{mol}$ for $\mathrm{H}_{2}$ and $\mathrm{D}_{2}$, respectively, the ZPE differences being the values of interest. The calculated values of $E_{\mathrm{vib}}^{M}$ and $E_{\mathrm{vib}}^{M \mathrm{H}_{y}}$ for each pure metal $M$ and hydride $M \mathrm{H}_{y}$ are compiled in the supplementary material (SM-B). All values are positive by definition, corresponding to a destabilizing effect. In the case of hydrides, it is well known that the high frequency optical branches are dominated by $\mathrm{H}-\mathrm{H}$ and $\mathrm{H}-M$ interactions and generally lie at considerably higher frequencies than the acoustic branches, because of the important mass difference between metal and hydrogen atoms. Therefore, the major contribution to $E_{\mathrm{vib}}^{M \mathcal{I}_{y}}$ arises from the vibration of $\mathcal{I}$ atom $(\mathrm{H}, \mathrm{D}$ or $\mathrm{T})$. 
As a linear decrease of the optical frequencies versus increasing metal hydrogen equilibrium distance $r$ was suggested in the literature [3, 1, 4], it is worth determining whether such a relationship can be also observed for the ZPE. The ZPE as a function of $r$ has been plotted in figure 3 for different hydrides with $y=0.5,1$ and 2 . It is possible to perform phonon calculations considering the same host metal $M$ for different hydride structures $M \mathrm{H}_{y}$, even if the structure is metastable. It allows us to study independently the effect of the crystal structure without changing the chemistry. Whatever the stoichiometry $y$, the data are quite scattered at a first glance and the $r$ parameter cannot be considered as a universal one. However, one can observe some general
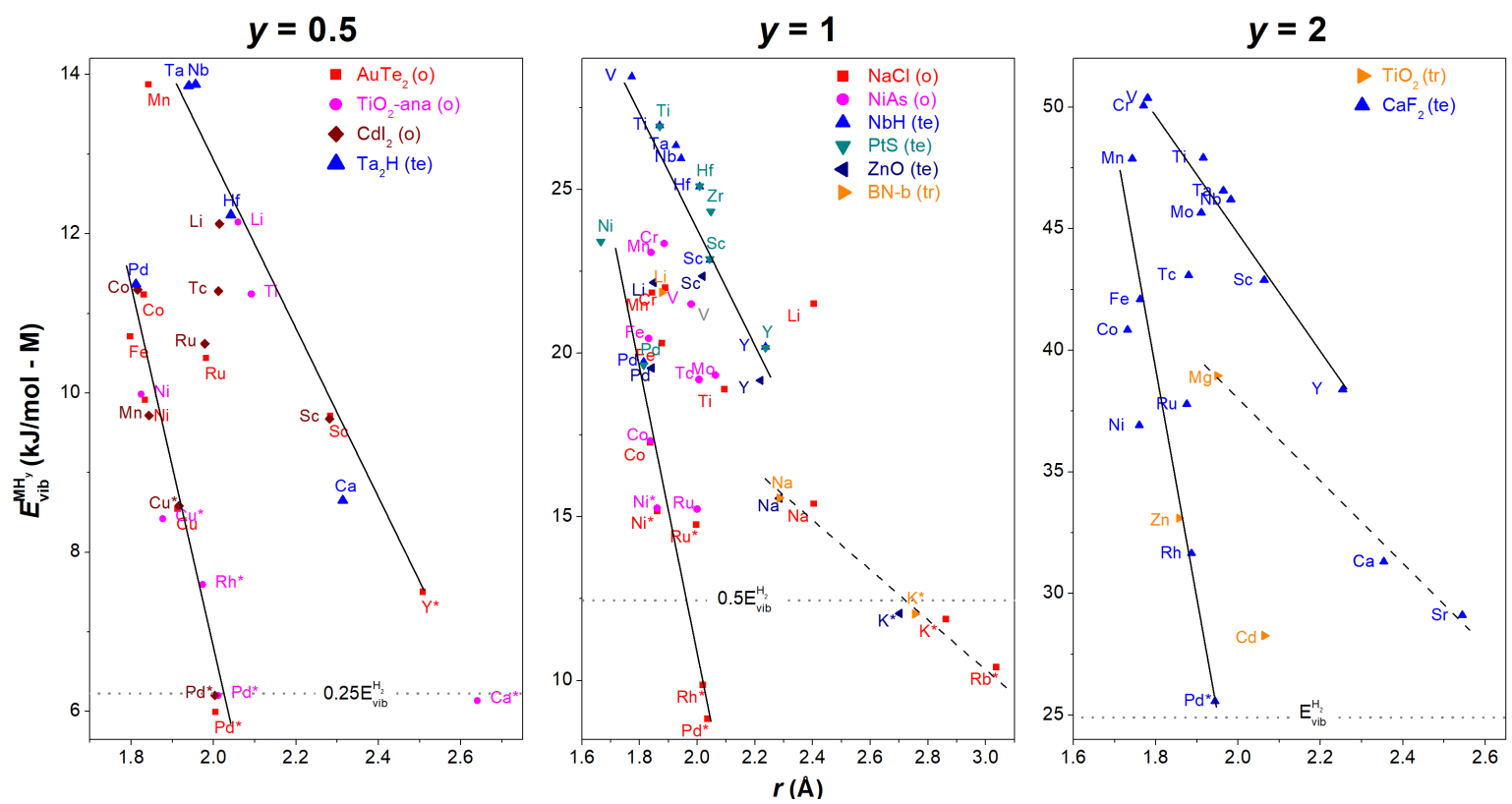

Figure 3. Calculated ZPE $E_{\mathrm{vib}}^{M \mathrm{H}_{y}}$ represented as functions of the average metalhydrogen distance $r$, for $y=0.5,1$ and 2. Only hydrides without imaginary frequency are represented. The o-site, te-site and tr-site hydrides are represented with redbased, blue-based and orange colors respectively. The asterisks point out the hydrides associated with negative $\Delta E^{\mathrm{H}, \mathrm{D}}$. The lines are guides to the eye indicating the general trend for hydrides with $M$ of similar electronic structure (straight lines: early and late transition metal hydrides; dashed lines: alkaline and alkaline earth metal hydrides). The horizontal dotted lines represent the ZPE value of the $\mathrm{H}_{2}$ molecule, weighted by $y / 2$.

trends. About, the influence of the type of coordination site, as the te-site prototypes have generally smaller $r$ than the o-sites, they are associated with stronger interatomic forces and therefore higher ZPE [36]. For a given host metal at a given composition, the calculated value of $r$ is systematically lower and the ZPE higher for the te-sites than for the o-sites. The distribution between the different elements highlights the influence of chemical bond nature and geometric effect, and could be analyzed by grouping elements with similar electronic structures. According to the hydrogen content $y, 4$ kinds of $M \mathrm{H}_{y}$ families are identified depending on $M$ : early and late $d$-elements, alkaline 
(for $y=1$ ) and alkaline earth metals (for $y=2$ ). For each family, because of a similar bonding nature associated to similar interatomic energies (e.g. ionic bond of the alkali hydrides), all the ZPE are aligned as shown by the straight and dashed lines in figure 3 . Several exceptions occur for hydrides of intermediate $d$-elements, such as Tc and Ru found between the early and late transition metals.

These results mean that the ZPE variation should be explained by taking into account both the chemical and geometrical nature of $M-\mathrm{H}$ bonds.

Besides, the formation enthalpy is affected by the ZPE. Two different situations may occur, as illustrated schematically in figure 4, depending, only, on the magnitude of the $\mathrm{ZPE}$ of the hydride $E_{\mathrm{vib}}^{M \mathrm{H}_{y}}$. If it is small compared to $E_{\mathrm{vib}}^{M}$ and $E_{\mathrm{vib}}^{\mathrm{H}_{2}}$, the ZPE contribution $\Delta E_{\mathrm{vib}}^{M \mathrm{H}_{y}}$ is negative and yields a stabilization of the hydride (s-case). On the contrary, if it is large, the resultant is positive and associated to a destabilization (d-case). Note that the contributions $E_{\mathrm{vib}}^{\mathrm{H}_{2}}$ is the same in all case and $E_{\mathrm{vib}}^{M}$ varies slightly from one $M$ to another. In the present study, the d-case was found predominant, with 94 out of 115

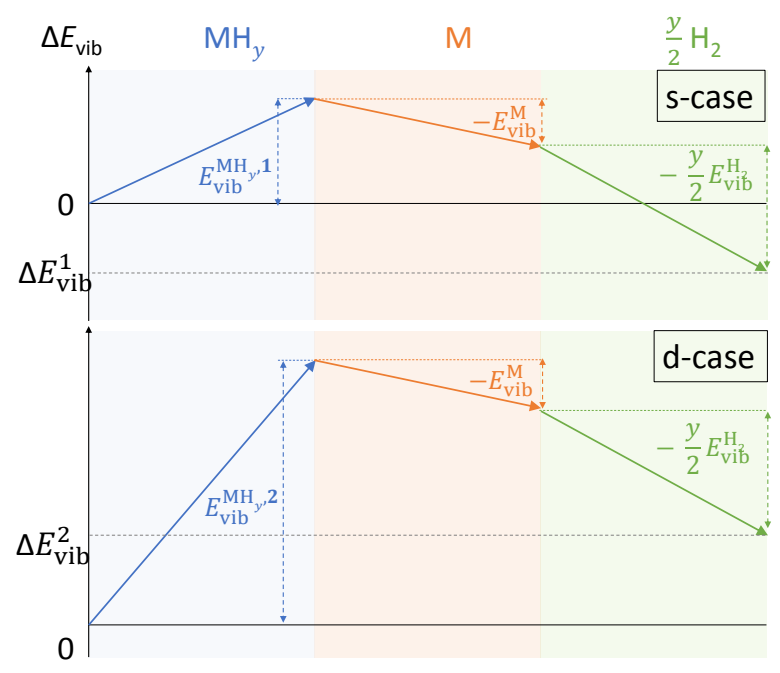

Figure 4. Schematic representation of the two cases: $\Delta E_{\mathrm{vib}}^{M \mathrm{H}_{y}}<0$ corresponding to a stabilization (s-case) and $\Delta E_{\mathrm{vib}}^{M \mathrm{H}_{y}}>0$ corresponding to a destabilization (d-case). The up arrow represents the positive contribution to $\Delta E_{\mathrm{vib}}^{M \mathrm{H}_{y}}$ and the down arrows the negative ones.

hydrides. The global set of values for $\Delta E_{\mathrm{vib}}^{M \mathrm{H}_{y}}$, compiled in the supplementary material, extends from -6.1 ( $\mathrm{PdH}$ in the $\mathrm{NaCl}$ prototype) to $+22.0 \mathrm{~kJ} / \mathrm{mol}\left(\mathrm{VH}_{2}\right.$ in the $\mathrm{CaF}_{2}$ prototype), representing a non negligible contribution to the total formation enthalpy.

\subsection{Isotope effect}

3.2.1. Calculations at zero kelvin The heavier the isotope is, the lower the ZPE is. The isotope effect arises thus from the competition between the lowering of the deuteride (tritide) frequencies on the one hand, and that of the $\mathrm{D}_{2}\left(\mathrm{~T}_{2}\right)$ molecule on the other hand, 
with respect to the corresponding hydride and the $\mathrm{H}_{2}$ molecule. Figure 5 illustrates schematically the competing contributions. It is thus quantified by the energy change when hydrogen substitutes its isotopes $\mathrm{D}$ or $\mathrm{T}$, which can be written as follows:

$$
\Delta E^{\mathrm{H}, \mathcal{I}}=\left[E_{\mathrm{vib}}^{M \mathrm{H}_{y}}-\frac{y}{2} E_{\mathrm{vib}}^{\mathrm{H}_{2}}\right]-\left[E_{\mathrm{vib}}^{M \mathcal{I}_{y}}-\frac{y}{2} E_{\mathrm{vib}}^{\mathcal{I}_{2}}\right] .
$$

with $\mathcal{I}=\mathrm{D}$ or $\mathrm{T}$.

Two cases can be distinguished according to the sign of $\Delta E^{\mathrm{H}, \mathcal{I}}$ :

- n-case: $\Delta E^{\mathrm{H}, \mathcal{I}}>0$, normal isotope effect at $0 \mathrm{~K}$ (concerns hydrides with high ZPE values). The deuteride (tritide) formation is favored with respect to that of the hydride (deuteride).

- i-case: $\Delta E^{\mathrm{H}, \mathcal{I}}<0$, inverse isotope effect at $0 \mathrm{~K}$ (concerns hydrides with low ZPE values). The hydride (deuteride) formation is favored with respect to that of the deuteride (tritide).

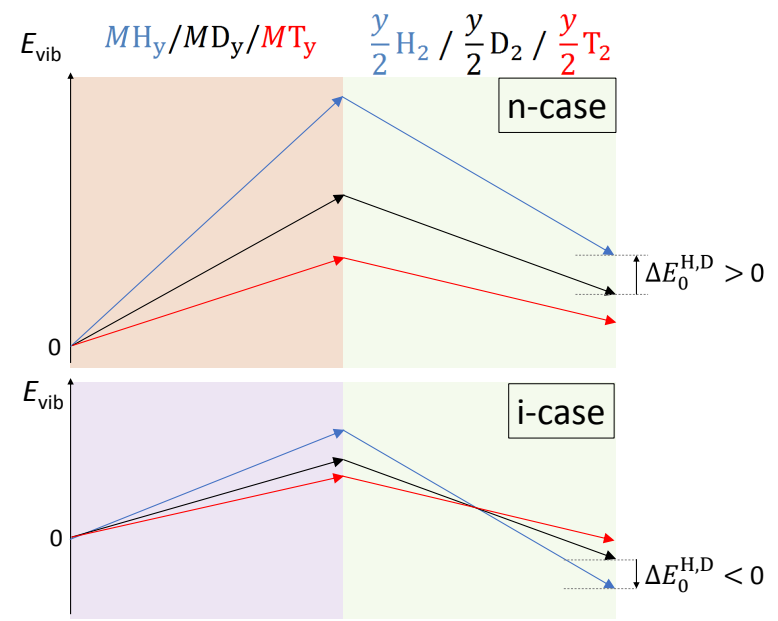

Figure 5. Schematic representation of the two types of isotope effects: $\Delta E^{\mathrm{H}, \mathcal{I}}<0$ corresponding to the inverse effect (i-case) and $\Delta E^{\mathrm{H}, \mathcal{I}}>0$ corresponding to the normal effect (n-case).

Figure 6 shows the calculated values of $\Delta E^{\mathcal{I}, \mathrm{D}}$ with $\mathcal{I}=\mathrm{H}$, $\mathrm{T}$ as function of $E_{\mathrm{vib}}^{M \mathrm{H}_{y}}-y E_{\mathrm{vib}}^{\mathrm{H}_{2}} / 2$. The dependence of the calculated $\Delta E^{\mathcal{I}, \mathrm{D}}$ values are well described by the linear fits, represented by the solid lines. Let's consider the Einstein model which supposes that all hydrogen atoms are independent harmonic oscillators, vibrating isotropically at the same frequency (1) and assuming that the contribution of the metallic atoms to the ZPE is negligible (2). In the following, the designation "Einstein model" includes hypotheses (1) and (2). This approach is often used in the literature to determine the ZPE of the deuteride $E_{\mathrm{vib}}^{M \mathrm{D}_{y}}$ from the measured value of $E_{\mathrm{vib}}^{M \mathrm{H}_{y}}$. The ZPE ratio can indeed be estimated by the inverse ratio of the squared isotope masses: $E_{\mathrm{vib}, 0}^{M \mathrm{H}_{y}} / E_{\mathrm{vib}, 0}^{M \mathcal{I}_{y}}=\sqrt{m_{\mathcal{I}}} / \sqrt{m_{\mathrm{H}}}$. The equations associated with the Einstein model, 
represented by the dotted lines in figure 6 , are as follow:

$$
\Delta E_{\text {Einstein }, 0}^{\mathcal{I}, \mathrm{D}}=\left(\sqrt{\frac{m_{\mathrm{H}}}{m_{\mathcal{I}}}}-\sqrt{\frac{m_{\mathrm{H}}}{m_{\mathrm{D}}}}\right) \times\left(E_{\mathrm{vib}, 0}^{M \mathrm{H}_{y}}-\frac{y}{2} E_{\mathrm{vib}, 0}^{\mathrm{H}_{2}}\right) .
$$

The slope is identical to that obtained by a linear regression of the phonon calculations. However, the X-intercept of the Einstein model is zero whereas for the present calculation it corresponds to the mean value of the metal atom contribution to the hydride ZPE, $E_{\mathrm{vib}, 0}^{M \text { in }} M \mathrm{H}_{y}=2.9 \mathrm{~kJ} / \mathrm{mol}$ in average, neglecting the coupling between the metal and hydrogen atom modes in the hydride. The isotope effect is then canceled when the hydrogen atom contribution to the hydride ZPE is equal to the molecule ZPE, weighted by $y / 2$ :

$$
E_{\text {vib }, 0}^{\mathrm{H} \text { in } M \mathrm{H}_{y}}=\frac{y}{2} E_{\text {vib }, 0}^{\mathrm{H}_{2}} .
$$

Moreover, in the case of the present phonon calculations, assuming that the vibration modes of the metal atoms are not modified by hydrogen insertion, it can be approximated that: $E_{\mathrm{vib}, 0}^{M \text { in } M \mathcal{I}_{y}}=E_{\mathrm{vib}, 0}^{M}$. Within this hypothesis, the transition from the n-case to the i-case occurs with the same criterion as the d-case to s-case transition, as in equation (5). One can thus consider that a destabilizing (stabilizing) vibration effect is generally associated with a normal (inverse) isotope effect.

3.2.2. Predictions at non zero temperatures At non zero temperatures, the Gibbs energy difference between the compounds $M \mathrm{H}_{y}$ and $M \mathcal{I}_{y}$ is considered instead of $\Delta E^{\mathrm{H}, \mathcal{I}}$. This temperature dependent value is designated by $\Delta G^{\mathrm{H}, \mathcal{I}}$ and defined as:

$$
\begin{aligned}
& \Delta G^{\mathrm{H}, \mathcal{I}}=\left[G_{\mathrm{vib}}^{M \mathrm{H}_{y}}-G_{\mathrm{vib}}^{M \mathcal{I}_{y}}\right]-\left[G^{\mathrm{H}_{2}}-G^{\mathcal{I}_{2}}\right] \\
& \Delta G^{\mathrm{H}, \mathcal{I}}=\Delta E^{\mathrm{H}, \mathcal{I}}-T \Delta S^{\mathrm{H}, \mathcal{I}},
\end{aligned}
$$

with $\Delta E^{\mathrm{H}, \mathcal{I}}$ and $\Delta S^{\mathrm{H}, \mathcal{I}}$ are temperature dependent.

The van't Hoff relationship, verified for formation pressures close to the atmospheric pressure, introduces the pressures of formation: $R T \ln \left(P_{\mathrm{H}_{2}} / P_{\mathcal{I}_{2}}\right)=\Delta \bar{G}^{\mathrm{H}, \mathcal{I}}$, with $\Delta \bar{G}^{\mathrm{H}, \mathcal{I}}$ defined with the partial enthalpy and entropy. When a hydride $M \mathrm{H}_{y}$ is formed from a lower hydride $M \mathrm{H}_{x}$, with $x<y$, the partial Gibbs energy of formation corresponds to the following reaction which does not involve the metal:

$$
M \mathrm{H}_{x}+\frac{y-x}{2} \mathrm{H}_{2} \rightleftharpoons M \mathrm{H}_{y} .
$$

In the present study, only the $\mathrm{Nb}-\mathrm{H}$ system is concerned. Indeed, formation pressures were measured for the couple $\mathrm{NbH}_{2} / \mathrm{NbD}_{2}$ corresponding to the following reaction: $\mathrm{NbH}+\mathrm{H}_{2} / 2 \rightleftharpoons \mathrm{NbH}_{2}$.

The values of $\Delta G^{\mathrm{H}, \mathrm{D}}$ as function of temperature were calculated for eleven stable hydride/deuteride couples for which experimental data are available: $\operatorname{Pd} \mathcal{I}, \mathrm{Ni} \mathcal{I}, \operatorname{Li} \mathcal{I}$, $\operatorname{Mo} \mathcal{I}, \operatorname{Cu} \mathcal{I}, \operatorname{Cr} \mathcal{I}, \operatorname{Sc} \mathcal{I}_{2}, \mathrm{YI}_{2}, \operatorname{Mg} \mathcal{I}_{2}, \operatorname{Ti} \mathcal{I}_{2}$ and $\mathrm{Nb} \mathcal{I}_{2}$, in their stable structure. The calculation results are presented in figure 7 . As the value of $G^{\mathrm{H}_{2}}-G^{\mathrm{D}_{2}}$ depends nearly linearly on the temperature between 0 and $1000 \mathrm{~K}$, we used the following linear regression 


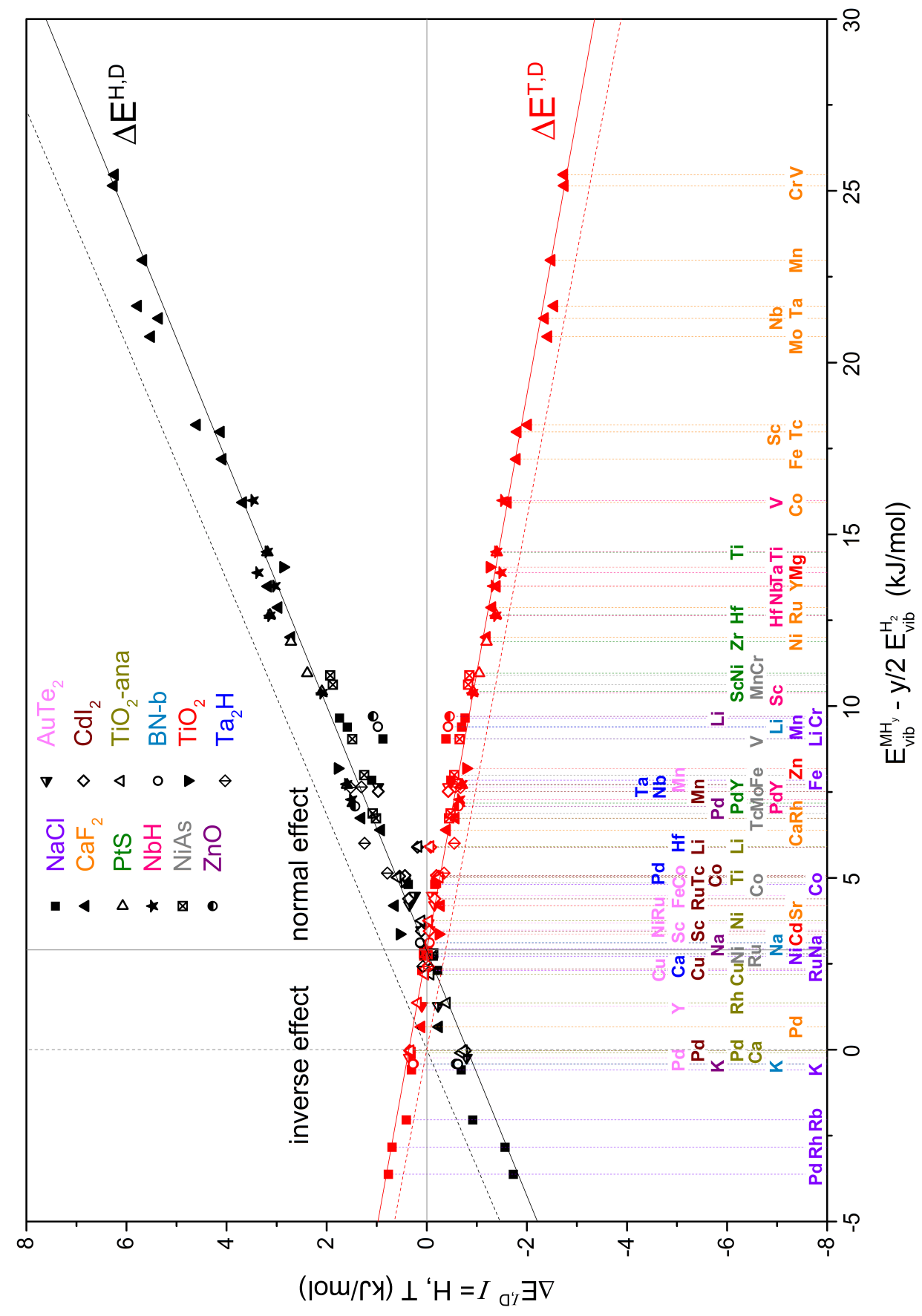

Figure 6. Calculated values of $\Delta E^{\mathcal{I}, \mathrm{D}}$ with $\mathcal{I}=\mathrm{H}$, $\mathrm{T}$ represented as a function of the calculated $E_{\mathrm{vib}}^{M \mathrm{H}_{y}}-\frac{y}{2} E_{\mathrm{vib}}^{\mathrm{H}_{2}}$. Only hydrides without imaginary frequency are represented. The hydrides whose $\Delta E^{\mathrm{H}, \mathrm{D}}$ are negative (positive) present an inverse (normal) isotope effect at $0 \mathrm{~K}$. The solid lines represent linear fits on the calculated values, to be compared with the Einstein model (dashed lines). 
$(\mathrm{R}=0.99986)$ to compute the represented points: $G^{\mathrm{H}_{2}}-G^{\mathrm{D}_{2}}=7.21002+T \cdot 1.431 \times$ $10^{-2} \mathrm{~kJ} / \mathrm{mol}$.

On the two graphs of figure 7, the Y-intercept corresponds to the value of $\Delta E_{0}^{\mathrm{H}, \mathrm{D}}$. It is positive for 9 of the $11 \mathrm{n}$-compounds. Only $\operatorname{Pd} \mathcal{I}$ and $\mathrm{Ni} \mathcal{I}$ present negative $\Delta E_{0}^{\mathrm{H}, \mathrm{D}}$ values (i-case). Besides, for each n-compound, the quantity $\Delta G^{\mathrm{H}, \mathrm{D}}$ changes its sign with increasing temperature. It expresses the transition towards an inverse effect. Simultaneously, the inverse isotope effect remains at high temperature for $\operatorname{Pd} \mathcal{I}$ and $\mathrm{Ni} \mathcal{I}$.
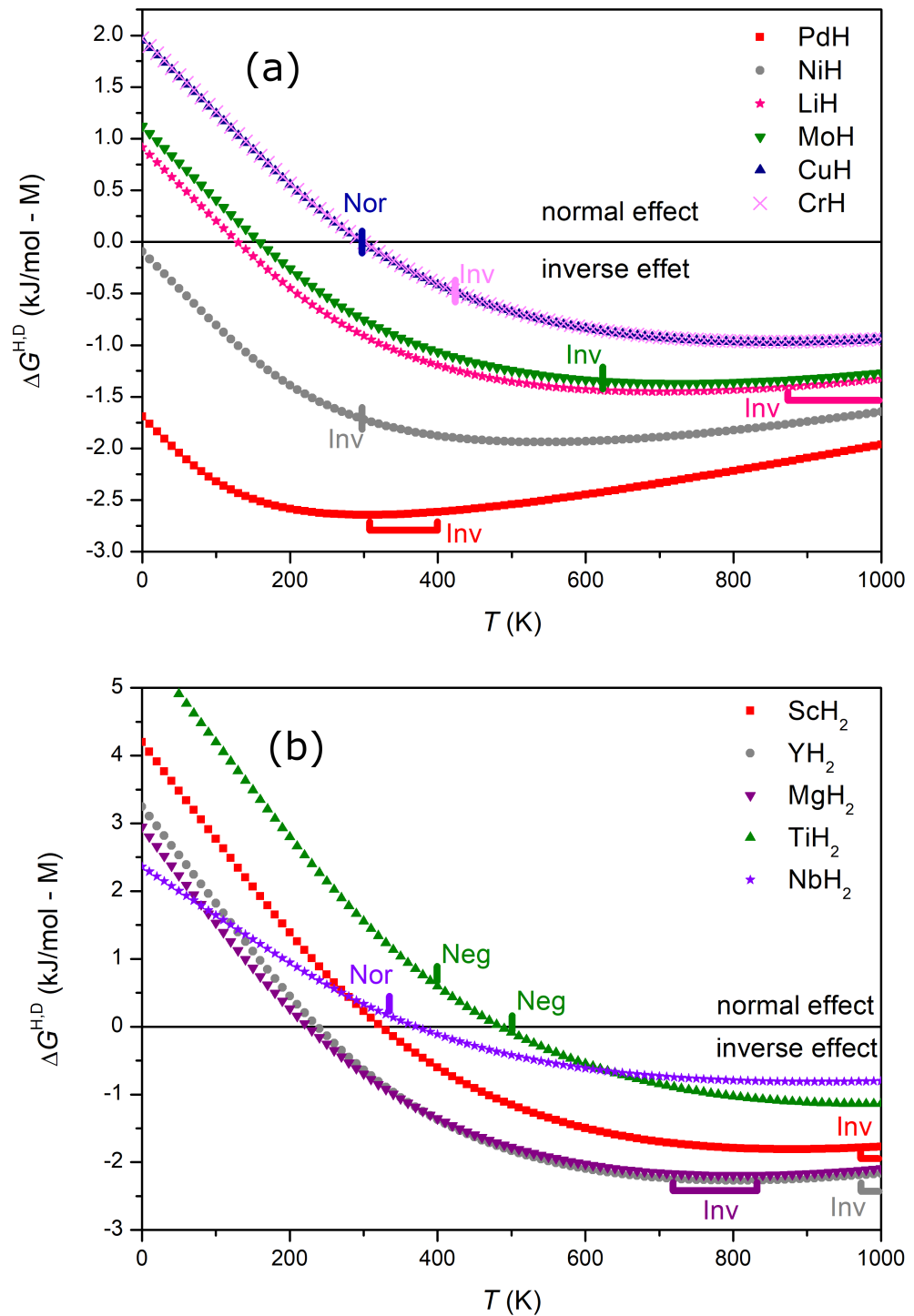

Figure 7. Variations of $\Delta G^{\mathrm{H}, \mathrm{D}}$ with temperature for a set of $M \mathcal{I}$ compounds (a) and $M \mathcal{I}_{2}$ (b) calculated with harmonic phonon calculations for the crystals and by estimating the contribution of the molecules by the linear regression of the JANAF table data [17]. The experimental results on the nature of isotope effect, inverse (Inv), normal (Nor) or negligible (Neg), are indicated at the temperature ranges corresponding to the experiments. 
Two types of behavior may occur, determined by the n- or i-case at $0 \mathrm{~K}$ :

- $n$-case: the normal isotope effect soften with increasing temperature until it reverses at a critical temperature $T_{\text {trans }}$, for which $\Delta G^{\mathrm{H}, \mathrm{D}}\left(T_{\text {trans }}\right)=0$.

- i-case: the inverse isotope effect remains at high temperature.

The hypothesis of the existence of a transition temperature $T_{\text {trans }}$ for the $\mathrm{n}$ compounds is therefore proposed. It arises from the entropy term associated with the molecules, which becomes dominant with increasing temperature:

$$
\Delta G^{\mathrm{H}, \mathrm{D}}=\left[E_{\mathrm{vib}}^{M \mathrm{H}_{y}}-E_{\mathrm{vib}}^{M \mathrm{D}_{y}}\right]-\left[E^{\mathrm{H}_{2}}-E^{\mathrm{D}_{2}}\right]-T\left[S_{\mathrm{vib}}^{M \mathrm{H}_{y}}-S_{\mathrm{vib}}^{M \mathrm{D}_{y}}\right]-T\left[S^{\mathrm{D}_{2}}-S^{\mathrm{H}_{2}}\right](9)
$$

A crossing of the Gibbs energies is expected for the n-case at $T_{\text {trans }}$, corresponding to a transition from normal to inverse isotope effect. Whereas for the i-case, no crossing is expected. Hence, a temperature increase favors the inverse isotope effect.

To our knowledge, a transition from normal to inverse isotope effect, for a given system, has neither been seen nor anticipated in the literature for binary hydrides. It may be difficult to show it experimentally by plateau pressure measurements because large temperature ranges inducing large pressure variations should be investigated with a high level of accuracy. If not the inversion itself, measurements should at least be able to demonstrate a decrease or an increase of the isotope effect as a function of temperature depending on the system. That would be a confirmation of our prediction. Additionally, note that such an inversion of the isotope effect has been experimentally observed for the hydrogenation (deuteriation) of the intermetallic compound $\mathrm{ErFe}_{2}$ [37], where the range of temperature is close to ambient conditions. It is highly probable that it is due to the same mechanism as described for binary hydrides.

The calculation results were compared to two types of experimental data: the formation pressure differences (1) and the formation enthalpy differences (2) between the hydride and deuteride.

(1) Equilibrium pressures have been reported in the literature for 12 hydride/deuteride couples, listed in table 3. These measurements reveal an inverse isotope effect for 8 compounds out of 12 . These observations are shown in figure 7 at the corresponding temperatures. They appeared in agreement with the calculated results. First, the inverse isotope effect measured for the six n-case compounds $\mathrm{YI}_{2}$, $\mathrm{Sc} \mathcal{I}_{2}, \operatorname{Mg} \mathcal{I}_{2}, \operatorname{Mo} \mathcal{I}, \operatorname{Cr} \mathcal{I}$ and Li $\mathcal{I}$ is in agreement with the calculated $T_{\text {trans }}$, which is below the temperature of the experiments.

Second, the cases of $\mathrm{Ti} \mathcal{I}_{2}, \mathrm{Cu} \mathcal{I}, \operatorname{Pd} \mathcal{I}, \mathrm{Ni} \mathcal{I}$ and $\mathrm{Nb} \mathcal{I}_{2}$ are discussed below. According to the calculations, $\mathrm{Ti} \mathcal{I}_{2}$ corresponds to the n-case. The measured formation pressures for $\mathrm{Ti} \mathcal{I}_{2}$ do not reveal any significant isotope effect. It may be due to the temperature of the measurements at 400 and $500 \mathrm{~K}$, which is close to the calculated value of $T_{\text {trans }}$ of $485 \mathrm{~K}$. It would be relevant to carry out experiments at other temperatures to check if the transition occurs. 
Table 3. Isotope effect pointed out by plateau pressure measurements on the hydride and deuteride.

\begin{tabular}{llll}
\hline$M \mathcal{I}_{y}$ (prototype) & Isotope effect (exp.) & $\mathrm{T}[\mathrm{K}]$ & ref. \\
\hline $\mathrm{Y} \mathcal{I}_{2}\left(\mathrm{CaF}_{2}\right)$ & inverse & 973 to 1273 & {$[38]$} \\
$\mathrm{Sc} \mathcal{I}_{2}\left(\mathrm{CaF}_{2}\right)$ & inverse & 973 to 1273 & {$[38]$} \\
$\mathrm{Ti}_{2}\left(\mathrm{CaF}_{2}\right)$ & negligible & 400 and 500 & {$[39]$} \\
$\mathrm{VI}_{2}\left(\mathrm{CaF}_{2}\right)$ & normal & 313 & {$[5]$} \\
$\mathrm{NbI} \mathcal{I}_{2}\left(\mathrm{CaF}_{2}\right)$ & normal & 334 & {$[5]$} \\
$\mathrm{MoI}(\mathrm{NiAs})$ & inverse & 623 & {$[40]$} \\
$\mathrm{Cr} \mathcal{I}(\mathrm{NiAs})$ & inverse & 423 & {$[41]$} \\
$\left.\mathrm{Mg} \mathcal{I}(\mathrm{TiO})_{2}\right)$ & inverse & 716 to 833 & {$[42]$} \\
$\mathrm{Cu} \mathcal{I}(\mathrm{ZnO})$ & normal & room temperature & {$[43,44]$} \\
$\mathrm{LiI}(\mathrm{NaCl})$ & inverse & 873 to 1123 & {$[45]$} \\
$\mathrm{Pd} \mathcal{I}(\mathrm{NaCl})$ & inverse & 308 to 398 & {$[46]$} \\
$\mathrm{Ni \mathcal {I }}(\mathrm{NaCl})$ & inverse & 298 & {$[47]$} \\
\hline
\end{tabular}

For the n-case $\mathrm{Cu} \mathcal{I}$ compound, the transition temperature is calculated at about $297 \mathrm{~K}$. The isotope effect should therefore be negligible at room temperature. However, the measured equilibrium pressures show a normal isotope effect at room temperature. This difference may be linked with the high formation pressure leading to a non negligible work of the pressure forces contributing to the enthalpy of formation. Such a contribution is also expected for the $\mathrm{Cr} \mathcal{I}, \mathrm{Mo} \mathcal{I}$ and $\mathrm{Cu} \mathcal{I}$ compounds whose equilibrium pressures are of the order of $10^{8}, 10^{9}$ and $10^{9} \mathrm{~Pa}$, respectively.

Moreover, for the $\mathrm{i}$-case compounds $\mathrm{Pd} \mathcal{I}$ and $\mathrm{Ni} \mathcal{I}$ the inverse isotope effect predicted at $0 \mathrm{~K}$ remains at high temperature, according to the calculations. In accordance, an inverse isotope effect is experimentally observed.

Finally, according to the calculations, $\mathrm{Nb} \mathcal{I}_{2}$ corresponds to the n-case, with a transition temperature predicted at $371 \mathrm{~K}$. In agreement, the isotope effect observed at $334 \mathrm{~K}$, which is below $T_{\text {trans }}$, is normal.

(2) When the formation enthalpies were measured for the hydride and the corresponding deuteride, the experimental value $\Delta E_{\exp }^{\mathrm{H}, \mathrm{D}}=\Delta E_{\mathrm{for}, \exp }^{M \mathrm{H}_{y}}-\Delta E_{\mathrm{for}, \exp }^{M \mathrm{D}_{y}}$ can be compared with the calculated values. The obtained experimental values are listed in the table 4 , together with the values computed at equivalent temperatures.

The agreement is satisfactory, with a maximum error of $1.5 \mathrm{~kJ} / \mathrm{mol}$, for $\mathrm{Zr} \mathcal{I}_{2}$. Concerning the alkaline metals, according to the experimental data, $\Delta E_{\text {exp }}^{\mathrm{H}, \mathrm{D}}$ is shifted towards negative values along the column with increasing the period. Thus, $\Delta E_{\exp }^{\mathrm{H}, \mathrm{D}}$ is positive for $\mathrm{Li}$, then becomes negative from $\mathrm{Na}$ and decreases up to $\mathrm{Rb}$. This trends is correctly reproduced by the calculations even with the simple harmonic approximation. Finally, one may note that the temperature dependence of $\Delta E^{\mathrm{H}, \mathrm{D}}$ has been found non-negligible for $\mathrm{Mg} \mathcal{I}_{2}$, changing from the positive value of $2.9 \mathrm{~kJ} / \mathrm{mol}$ at $0 \mathrm{~K}$ to a negative value of $-2.0 \mathrm{~kJ} / \mathrm{mol}$ at $800 \mathrm{~K}$, in agreement with the negative experimental value. 
Table 4. Experimental values of $\Delta E_{\text {exp }}^{\mathrm{H}, \mathrm{D}}$ compared to calculated one $\Delta E_{\text {calc }}^{\mathrm{H}, \mathrm{D}}$ at equivalent temperatures and the values computed at $0 \mathrm{~K} \Delta E_{0, \text { calc }}^{\mathrm{H}, \mathrm{D}}$. The values are given in $\mathrm{kJ} / \mathrm{mol}$.

\begin{tabular}{lrrrll}
\hline$M \mathcal{I}_{y}$ (prototype) & $\Delta E_{0, \text { calc }}^{\mathrm{H}, \mathrm{D}}$ & $\Delta E_{\text {calc }}^{\mathrm{H}, \mathrm{D}}(T[\mathrm{~K}])$ & $\Delta E_{\text {exp }}^{\mathrm{H}, \mathrm{D}}$ & method $(T[\mathrm{~K}])$ & ref. \\
\hline $\mathrm{YI}_{2}\left(\mathrm{CaF}_{2}\right)$ & 3.2 & $3.2(0)$ & 3.3 & integration of exp. $C_{p}(0)$ & {$[42]$} \\
$\mathrm{Zr \mathcal {I } _ { 2 }}\left(\mathrm{CaF}_{2}\right)$ & 4.8 & $4.0(300)$ & 5.5 & calorimetry $(298)$ & {$[48]$} \\
$\mathrm{Mg} \mathcal{I}_{2}\left(\mathrm{TiO}_{2}\right)$ & 2.9 & $-2.0(800)$ & -1.3 & van't Hoff $(716$ to 833$)$ & {$[42]$} \\
$\mathrm{Li} \mathcal{I}(\mathrm{NaCl})$ & 0.9 & $0.2(300)$ & 0.5 & calorimetry $(298)$ & {$[49]$} \\
$\mathrm{Na \mathcal {I }}(\mathrm{NaCl})$ & 0.1 & $0.1(300)$ & -0.6 & calorimetry $(298)$ & {$[49]$} \\
$\mathrm{KI}(\mathrm{NaCl})$ & -0.7 & $-1.6(300)$ & -2.4 & calorimetry $(298)$ & {$[49]$} \\
$\mathrm{RbI}(\mathrm{NaCl})$ & -0.9 & $-1.9(300)$ & -3.0 & van't Hoff $(?)$ & {$[50]$} \\
$\mathrm{Pd} \mathcal{I}(\mathrm{NaCl})$ & -1.7 & $-2.6(300)$ & -1.8 & calorimetry $(298)$ & {$[51]$} \\
\hline
\end{tabular}

The differences between calculated and experimental values may arise from different sources. First, hypotheses considered in the DFT introduce an error, by assuming a perfectly ordered atomic lattice, without any defect. Second, phonon calculations are based on simplifying assumptions: the anharmonic contribution is neglected and the interatomic forces and lattice parameters are assumed to be constant for the hydride, deuteride, and tritide. Third, when a partial enthalpy of formation is reported, it has to be integrated in order to be compared with the calculated integral quantities. Integration is achieved by neglecting the presence of solid solution domains. A shift may therefore arise, particularly when the experiment is carried out at high temperature. However, the agreement between theoretical and experimental results should be emphasized, given the simplicity of the model.

\section{Conclusions}

The phonon calculations provided a consequent hydride database, which allowed to highlight the following trends. The ZPE values depend on both geometric and chemical arguments, where inside a $M \mathrm{H}_{y}$ family with a similar electronic structure of $M$, the ZPE decreases as the $M-\mathrm{H}$ distance $r$ increases. The calculated ZPE has been systematically used to correct the formation enthalpy of a large binary hydride database in a previous work [7]. Its value determines the type of isotope effect at $0 \mathrm{~K}$ divided into normal (n) and inverse (i) cases. The n-case appeared predominant. The n-case (respectively i-case) is expected to be associated with a stabilizing (respectively destabilizing) ZPE contribution to the enthalpy of formation. Observation of 8 on the 11 studied systems have been reported in good agreement with the present model, described simply using the harmonic approximation. Moreover, it is predicted that for all the n-systems, the normal isotope effect switches to inverse effect at high temperature, which has never been observed before. Experiments are planned to investigate more into detail the 
temperature dependence of the isotope effect for different metal-hydrogen systems.

\section{Acknowledgments}

This work has benefited from a French government grant managed by ANR within the framework of the national program Investments for the Future ANR-11-LABX-022-01. DFT calculations were performed using HPC resources from GENCI-CINES (Grant 2017-96175). The authors gratefully acknowledge SR16000 supercomputing resources from the CCMS of the IMR, Tohoku University, Japan.

\section{Appendix A. Supplementary materials}

List of the 115 considered hydrides

\section{Appendix B. Supplementary materials}

Calculated values of $E_{\mathrm{vib}}^{M}$ and $E_{\mathrm{vib}}^{M \mathrm{H}_{y}}$ for each pure metal $M$ and hydride $M \mathrm{H}_{y}$

\section{References}

[1] D. Ross, P. Martin, W. Oates, and R. K. Bakhsh. Inelastic Neutron Scattering Measurements of Optical Vibration Frequency Distributions in Hydrogen-Metal Systems. Z. Phys. Chem., 114(114):221-230, 1979.

[2] E. Fermi. Sul moto dei neutroni nelle sostanze idrogenate. Ric. Sci., 7:13, 1936.

[3] M. Sakamoto. Studies of Hydrogen Vibrations in Transition Metal Hydrides by Thermal Neutron Transmissions. J. Phys. Soc. Japan, 19(10):1862-1866, 1964.

[4] Y. Fukai and H. Sugimoto. On the optical-mode excitation energy interstitial hydrogen in metals. J. Phys. F: Met. Phys., 11(7):L137, 1981.

[5] R. H. Wiswall and J. J. Reilly. Inverse Hydrogen Isotope Effects in Some Metal Hydride Systems. Inorg. Chem., 11(7):1691-1696, 1972.

[6] G. Sandrock. Applications of Hydrides, pages 253-280. Springer Netherlands, Dordrecht, 1995.

[7] N. Bourgeois, J.-C. Crivello, P. Cenedese, and J.-M. Joubert. Systematic first-principles study of binary metal hydrides. ACS Comb.Sci., 19(8):513-523, 2017.

[8] M. T. Dove, editor. Introduction to Lattice Dynamics. Cambridge University Press, Cambridge, 1993.

[9] K. Parlinski, Z. Q. Li, and Y. Kawazoe. First-Principles determination of the soft mode in cubic $\mathrm{ZrO}_{2}$. Phys. Rev. Lett., 78(21):4063-4066, 1997.

[10] W. Kohn and L. J. Sham. Self-consistent equations including exchange and correlation effects. Phys. Rev., 140:A1133-A1138, Nov 1965.

[11] A Togo and I Tanaka. First principles phonon calculations in materials science. Scr. Mater., 108:1-5, Nov 2015.

[12] G. Kresse and J. Furthmüller. Efficient iterative schemes for ab initio total-energy calculations using a plane-wave basis set. Phys. Rev. B, 54(16):11169-11186, Oct 1996.

[13] G. Kresse and D. Joubert. From ultrasoft pseudopotentials to the projector augmented-wave method. Phys. Rev. B, 59(3):1758-1775, 1999.

[14] J.P. Perdew, M. Ernzerhof, and K. Burke. Rationale for mixing exact exchange with density functional approximations. J. Chem. Phys., 105(22):9982-9985, 1996. 
[15] J.P. Perdew, K. Burke, and M. Ernzerhof. Generalized Gradient Approximation Made Simple. Phys. Rev. Lett., 78(7):1396, 1997.

[16] H.J. Monkhorst and J.D. Pack. Special points for Brillouin-zone integrations. Phys. Rev. B, 13(12):5188-5192, 1976.

[17] M. W. Chase, editor. NIST-JANAF Thermochemical Tables. American Institute of Physics for the National Institute of Standards and Technology, Washington, D.C., 1998.

[18] J. L. Verble, J. L. Warren, and J. L. Yarnell. Lattice dynamics of lithium hydride. Phys. Rev., 168:980-989, Apr 1968.

[19] A.I. Kolesnikov, V.E. Antonov, V.S. Efimchenko, G. Granroth, S.N. Klyamkin, A.V. Levchenko, M.K. Sakharov, and Y. Ren. Neutron spectroscopy of magnesium dihydride. J. Alloys Compd., 509, Supplement 2:S599 - S603, 2011. Proceedings of the 12th International Symposium on Metal-Hydrogen Systems, Fundamentals and Applications (MH2010).

[20] I. Errea, M. Calandra, and F. Mauri. Anharmonic free energies and phonon dispersions from the stochastic self-consistent harmonic approximation: Application to platinum and palladium hydrides. Phys. Rev. B, 89:064302, 2014.

[21] V.E. Antonov, T.E. Antonova, V.K. Fedotov, T. Hansen, A.I. Kolesnikov, and A.S. Ivanov. Neutron scattering studies of $\gamma$-CoH. J. Alloys Compd., 404-406:73-76, 2005.

[22] V.E. Antonov, A.I. Beskrovnyy, V.K. Fedotov, A.S. Ivanov, S.S. Khasanov, A.I. Kolesnikov, M.K. Sakharov, I.L. Sashin, and M. Tkacz. Crystal structure and lattice dynamics of chromium hydrides. J. Alloys Compd., 430(12):22 - 28, 2007.

[23] B. Dorner, I.T. Belash, E.L. Bokhenkov, E.G. Ponyatovsky, V.E. Antonov, and L.N. Pronina. Inelastic incoherent neutron scattering spectra from fcc $\mathrm{NiH}_{1.05}$, hcp $\mathrm{CrH}_{1.0}$ and hcp $\mathrm{MoH}_{1.2}$ at 15 K. Solid State Commun., 69(2):121-124, 1989.

[24] P. A. Korzhavyi, I. L. Soroka, E. I. Isaev, C. Lilja, and B. Johansson. Exploring monovalent copper compounds with oxygen and hydrogen. Proc. Natl. Acad. Sci. U. S. A., 109(3):686-689, 2012.

[25] K. Cornell, H. Wipf, V.E. Antonov, T.E. Antonova, A.I. Kolesnikov, E.G. Ponyatovsky, and B. Dorner. The inelastic neutron scattering spectrum of dhcp iron hydride. Pol. J. Chem., 71(12):1792-1796, 1997.

[26] M.H. Brodsky and E. Burstein. Infrared lattice vibrations of single crystal lithium hydride and some of its isotopic derivations. J. Phys. Chem. Solids, 28(9):1655 - 1668, 1967.

[27] V.E. Antonov, K. Cornell, B. Dorner, V.K. Fedotov, G. Grosse, A.I. Kolesnikov, F.E. Wagner, and H. Wipf. Neutron spectroscopy of $\gamma$ manganese hydride. Solid State Commun., 113(10):569$572,2000$.

[28] D. Richter. Localized mode energies and hydrogen potential in refractory metals. J. Less-Common Met., 89(2):293-306, 1983.

[29] A.I. Kolesnikov, I. Natkaniec, V.E. Antonov, I.T. Belash, V.K. Fedotov, J. Krawczyk, J. Mayer, and E.G. Ponyatovsky. Neutron spectroscopy of $\mathrm{MnH}_{0.86}, \mathrm{NiH}_{1.05}, \mathrm{PdH}_{0.99}$ and harmonic behaviour of their optical phonons. Phys. B (Amsterdam, Neth.), 174(1):257-261, 1991.

[30] R. Yamada, N. Watanabe, K. Sato, H. Asano, and M. Hirabayashi. Localized modes in tantalum hydrides studied by neutron inelastic scattering. J. Phys. Soc. Jpn., 41(1):85-90, 1976.

[31] S. Ikeda, N. Watanabe, and K. Kai. Crystal analyser TOF spectrometer (CAT). Physica B+C, 120(1):131-135, 1983.

[32] J. J. Rush, N. F. Berk, A. Magerl, J. M. Rowe, and J. L. Provo. Anomalous vibrations of hydrogen isotopes in $\beta$-phase vanadium hydride. Phys. Rev. B, 37:7901-7903, 1988.

[33] J. J. Rush, H. E. Flotow, D. W. Connor, and C. L. Thaper. Vibration spectra of yttrium and uranium hydrides by the inelastic scattering of cold neutrons. J. Chem. Phys., 45(10):3817-3825, 1966.

[34] J. G. Couch, O. K. Harling, and Lavern C. Clune. Structure in the neutron scattering spectra of zirconium hydride. Phys. Rev. B, 4:2675-2681, 1971.

[35] K.K. Irikura. Experimental vibrational zero-point energies: Diatomic molecules. J. Phys. Chem. 
Ref. Data, 36(2):389-397, 2007.

[36] G. Sicking. Isotope Effects in Metal-Hydrogen Systems. J. Less Common Met., 101:169-190, 1984.

[37] T. B. Flanagan, J. D. Clewley, N. B. Mason, H. S. Chung. Thermodynamics of the $\mathrm{ErFe}_{2}-\mathrm{H}(\mathrm{D})$ system. J. Less Common Met., 130:309-318, 1987.

[38] G.M. Begun, J.F. Land, and J.T. Bell. High temperature equilibrium measurements of the yttriumhydrogen isotope (H2, D2, T2) systems. J. Chem. Phys., 72(5):2959-2966, 1980.

[39] R. M. Haag and F. J. Shipko. The Titanium-Hydrogen System. J. Am. Chem. Soc., 78(20):5155$5159,1956$.

[40] VE Antonov, AI Latynin, and M Tkacz. T-P phase diagrams and isotope effects in the Mo-H/D systems. J. Phys.: Condens. Matter, 16(46):8387-8398, 2004.

[41] B. Baranowski, K. Bojarski, and M. Tkacz. Cr-H and Cr-D Systems in the High Pressure Region. In Proc. Fourth Int. Conf. High Press. Physico-Chemical Soc. Japan, Kyoto., pages 577-579, Kyoto, 1975. The Physico-Chemical Society of Japan.

[42] J. F. Stampfer Jr., C. E. Holley, and J. F. Suttle. The magnesium-hydrogen system. J. Am. Chem. Soc., 82(14):3504-3508, 1960.

[43] M. Tkacz and R. Burtovyy. Decomposition of the hexagonal copper hydride at high pressure. Solid State Commun., 132(1):37-41, 2004.

[44] M. Tkacz and R. Burtovyy. Isotope effect in a $\mathrm{CuH}(\mathrm{D})$ system with hexagonal hydride phase. J. Alloys Compd., 404-406:368-371, 2005.

[45] E. Veleckis. Decomposition pressures in the $(\alpha+\beta)$ fields of the Li-LiH, Li-LiD, and Li-LiT systems. J. Nucl. Mater., 79:20-27, 1979.

[46] R. Lässer and K. H. Klatt. Solubility of Hydrogen Isotopes in Palladium. Phys. Rev. B, 28:748758, Jul 1983.

[47] M. Tkacz. Enthalpies of formation and decomposition of nickel hydride and nickel deuteride derived from (p,c,t) relationships. J. Chem. Thermodyn., 33(8):891 - 897, 2001.

[48] D.R. Fredrickson, R.L. Nuttall, H.E. Flotow, and W.N. Hubbard. The Enthalpies of Formation of Zirconium Dihydride and Zirconium Dideuteride. J. Phys. Chem., 67(7):1506-1509, 1963.

[49] S.R. Gunn and L.G. Green. The Heats of Formation at $25^{\circ}$ of the Crystalline Hydrides and Deuterides and Aqueous Hydroxides of Lithium, Sodium and Potassium. J. Am. Chem. Soc., 80(18):4782-4786, 1958.

[50] L Hackspill and A Borocco. The Preparation of Alkali Deuterides and a Comparison of their Dissociation Pressures with those of the Corresponding Hydrides. Bull. Soc. Chim. Fr., 6:91103, 1939.

[51] Ted B. Flanagan, W. Luo, and J.D. Clewley. Calorimetric enthalpies of absorption and desorption

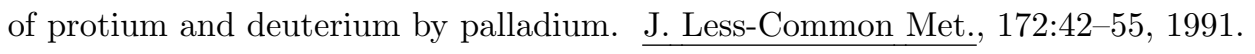

